\title{
A Proof of Syntactic Incompleteness of the Second-Order Categorical Arithmetic
}

\author{
Giuseppe Raguní \\ UCAM-Catholic University of Murcia, Murcia, Spain \\ Email: graguni@ucam.edu
}

How to cite this paper: Raguní, G. (2017) A Proof of Syntactic Incompleteness of the Second-Order Categorical Arithmetic. Open Access Library Journal, 4: e3969 https://doi.org/10.4236/oalib.1103969

Received: September 24, 2017

Accepted: October 23, 2017

Published: October 26, 2017

Copyright $\odot 2017$ by author and Open Access Library Inc.

This work is licensed under the Creative Commons Attribution International License (CC BY 4.0).

http://creativecommons.org/licenses/by/4.0/

\begin{abstract}
Actually, Arithmetic is considered as syntactically incomplete. However, there are different types of arithmetical theories. One of the most important is the second-order Categorical Arithmetic $(A R)$, which interprets the principle of induction with the so-called full semantics. Now, whoever concluded that $A R$ is sintactically (or semantically, since categoricity implies equivalence of the two types of completeness) incomplete? Since this theory is not effectively axiomatizable, the incompleteness Theorems cannot be applied to it. Nor is it legitimate to assert that the undecidability of the statements is generally kept in passing from a certain theory (such as $P A$ ) to another that includes it (such as $A R$ ). Of course, although the language of $A R$ is semantically incomplete, this fact does not imply that the same $A R$ is semantically/sintactically incomplete. Pending a response to the previous question, this paper aims to present a proof of the syntactic/semantical incompleteness of $A R$, by examples based on the different modes of representation (i.e. codes) of the natural numbers in computation.
\end{abstract}

\section{Subject Areas}

Mathematical Logic and Foundation of Mathematics

\section{Keywords}

Second order, Arithmetic, Categoricity, Incompleteness,

Undecidability, Codes, Algorithmic Randomness

\section{Introduction}

The formal Peano's Arithmetic $(P A)$ and the second-order Categorical Arithmetic $(A R)$, differ only by the interpretation of the principle of induction: 


$$
\forall p([p(0) \wedge \forall x(p(x) \rightarrow p(s(x)))] \rightarrow \forall x(p(x)))
$$

where $s(x)$ is the succesor of $x$. In $P A, p$ indicates any property that is expressible in the theory with a formula with (at least) one free variable, called $x$. In $A R, p$ just indicates any unambiguous property of $x$. This different interpretation has important consequences.

In set-theoretical language (formalizable in any formal Set Theory, as $N B G$, $Z F$ or $M K$ ) we render the natural numbers as sets and make correspond a subset of natural numbers to each unambiguous property, so getting in the case of $P A$ :

$$
\forall A \in P(U)_{1}([0 \in A \wedge \forall x \in U(x \in A \rightarrow x+1 \in A)] \rightarrow A=U)
$$

where $U$ is the set-universe of the model (called $N$ in the standard model of the natural numbers) and $P(U)_{1}$ the set of all subsets of $U$ whose elements satisfy a property that is expressible with a formula with (at least) one free variable. $P(U)_{1}$ turns out to be denumerable [1], even effectively denumerable ${ }^{1}$. Consequently, in $P A$ the principle of induction is an axiomatic schema that generates an effectively denumerable number of axioms. Therefore, the theory is effectively axiomatizable $^{2}$ and the incompleteness Theorems can be applied to it.

On the other hand, for $A R$ we get a similar set-expression for the induction but instead of $P(U)_{1}$ we have just $P(U)$, the set of all subsets of $U$. Now, if $U$ is infinite (and this will be confirmed by the fact that $U$ is equal or isomorphic to $N) P(U)$ has a non-denumerable cardinality. Therefore, in this case the inductive axiomatic schema generates a non-denumerable number of axioms and the theory is not effectively axiomatizable: it does not satisfy the hypotheses of the incompleteness Theorems ${ }^{3}$.

Besides, the quantification over all subsets of the set-universe (briefly called full second-order semantics) allows to demonstrate the categoricity of $A R$, i.e. that this theory has only one model (the standard one), up to isomorphism (Dedekind's Theorem $)^{4}$.

\section{Is the Second Order Categorical Arithmetic (AR) Sintactically/Semantically Incomplete?}

Although the incompleteness Theorems do not apply to $A R$, it could be thought that the undecidable statements of $P A$, or part of them, continue to be undecidable in $A R$. But this is not guaranteed.

Making use also of non-effective deductive methods, $A R$ is capable to deduce a non-denumerable number of theorems, to which hence is impossible to assign an univocal code (like a gödelian code), except for an insignificant number of

\footnotetext{
${ }^{1}$ Throughout the paper, we consider as valid the Church-Turing Thesis, so using always "effectively" rather than "recursively".

${ }^{2}$ Originally, effective axiomatizability is defined as decidability for the axioms. However, due to the Craig's Theorem, at first order this definition can be made lighter by requiring just effective denumerability [2].

${ }^{3}$ Evidently, the ambigous terminology of "strong enough arithmetical theory" here leads to error.

${ }^{4}$ The addition of any comprehension axioms in $A R$ invalidates the categoricity [3].
} 
cases; the same is true for the proofs. Thus, no similar strategy to that one used by Gödel to identify undecidable statements can be successful in $A R$. Here the Gödel's famous statement $(G)$, undecidable in $P A$, still means "no gödelian code of a proof of myself exists" but no longer "I'm not a theorem", since not every proof has a gödelian code (those that have it, conversely, are insignificant). Nothing prohibits that $G$ can be demonstrated in $A R$ (here meaning "I am not provable in $P A$ ") by one of the new inductive inferences. Analogous considerations apply to the undecidable propositions considered by the Second incompleteness Theorem.

The full semantics contained in the principle of induction, admitting the predicability of any property that is expressible by the set theory language, increases dramatically the power and deductive skills of the language: references to meta-mathematical concepts, to the truth, to other mathematical theories and their properties, etc., are possible with the only condition of unambiguity from a meta-mathematical point of view. And the mere use of the concept of truth to deduce, seems enough to make questionable the syntactic incompleteness of the theory.

Due to its categoricity, syntactic and semantical completeness ${ }^{5}$ are equivalent for $A R$. Anyhow, although the language used in $A R$ is not semantically complete $^{6}$, this does not imply that $A R$ is semantically (or syntactically) incomplete. To show it with an example, let consider the theory whose axioms are obtained by adding all sentences true in the standard model to the axioms of $A R$. We get a syntactically complete and still categoric system: thus, also semantically complete. But due to the categoricity, its language continues to be semantically incomplete.

By the First incompleteness Theorems it is easy to show that the valid formulas of $A R$ cannot be effectively deduced (e.g. [4] and [5]). This kind of semantical incompleteness is called sometimes inherent [5] or essential [6], but these adjectives are not appropriate because, naturally, it does not imply the genuine semantical incompleteness: it does not forbid that the valid formulas of $A R$ can be deduced.

Actually, the syntactic/semantical incompleteness of $A R$ is yet to be proven and the only hint for a proof is based on the difference between the concepts of syntactic implication and truth [7].

\section{Examples of Undecidable Statements in $A R$}

In computation, we assign a symbolic code to each natural number and, according to it, define the operation rules. The most common codes are those logarithmic, in base: $10,2,32,64 \cdots$; but, of course, their theoretical number is infi-

\footnotetext{
${ }^{5}$ Where for semantically complete language (or, in a different case, theory) we understand a system always interpretable if consistent (or, that is equivalent, in which all the valid formulas are deducible).

${ }^{6}$ By contradiction, we could apply the Löwenheim-Skolem (or Semantical Compactness) Theorem to conclude that $A R$ is not categorical.
} 
nite. As a classic, non-conventional example, we cite the representation (which we will call explicit) via one symbol (as “0” or “/”) repeated $n+1$ times: “/” for 0 , "//" for 1, etc. In principle, we are free to use arbitrarily irregular codes; for example, one in which, starting from a certain $n$ (or, more generally, for every element of a certain subset of natural numbers) new symbols of representation and/or operation rules are utilized [8]. The only thing that matters, from a logical point of view, is that then we are capable to define successfully the arithmetical operations, anyhow complicated they could be. The choice of a code rather than another determines differences in features that usually are not interesting for the Arithmetic: if we maintain this convention, models that use different codes are anyway isomorphic. But the full semantics allows to use such features to define unambiguous properties for natural numbers. A statement that mentions these properties has high probability of being unprovable.

Examples are statements such as "every $n$ is encoded by a chain of $n+1$ symbols" or "every $n$ is encoded by a chain containing at most two different symbols". In fact they, nor their negated, can be theorems of $A R$ : there exist interpretations of $A R$ in which they are "true" and others in which they are "false" . This does not preclude that they are isomorphic because this new "truth", having no properly arithmetical interest, can be excluded in order to distinguish different models (so we are using the quotes). It is quite easy to build other undecidable statements that refer to the code.

More interesting examples of undecidability are obtained taking into account the concept of algorithmic randomness [9] [10] [11], which, in fact, refers to the symbolic strings. If-following a Chaitin's (even tacit) convention-we define that a natural number is random if the string that represents it (i.e. its code) is random, then we have that the statement "infinite random natural numbers exist" is undecidable. In fact it is "true" for the usual logarithmic codes of any base, but for example "false" for the explicit code. In fact, starting from a sufficiently large $n$, the string " $/ / \cdots$ ( $n$ times) $\cdots /$ ", and all the subsequent ones, are efficiently compressible (using, for example, any logarithmic code), whatever the degree of efficiency is agreed upon. Indeed, Chaitin's version of the First incompleteness Theorem [12] does not prove that there is "randomness in Arithmetic" , but that no arithmetical theory that satisfies the hypotheses of the theorem can prove that a long enough symbolic string is random, if it is so.

\section{Consistent Extensions of $\boldsymbol{A R}$ without Interpretations}

With the introduction of properties that mention the code, isomorphic models of $A R$ can be distinguished on the basis of "truths" that usually are not considered arithmetical and, therefore, capable to invalidate the isomorphism. If, however, we decide to include the code between the properties that characterize

\footnotetext{
${ }^{7}$ One can also observe that in the principle of induction (the only one that could infer such statements), the satisfaction of the property for $n$ does not imply satisfaction for $n+1$, due to the aforementioned arbitrariness of the codes.

${ }^{8}$ Against the assertions of the same Chaitin, see [13] and [14].
} 
a model, then an above described undecidable statement, considered as a new axiom, generates a theory that admits only specific models, i.e. equipped with a particular type of code. For example, if we choose as a new axiom the statement "every $n$ is encoded by a chain containing at most two different symbols", the new theory admits as specific models: the one who uses the explicit code, the logarithmic-base 2 one and infinite others, together with all the isomorphic ones to them. The same thing happens with the statement "infinite random natural numbers exist".

However, even if you include the code between the properties that define a model, it is possible to show undecidable statements that are not satisfiable by any model. An interesting example is the statement "the code of every $n$ is a random sequence" (or "every $n$ is random", following the convention above). Not only it is false in every model with a conventional code, but any attempt to build a special model in which it is true has to fail, because it would require you to be able to identify random numbers arbitrarily large, in violation of Chaitin's version of the First incompleteness Theorem ${ }^{9}$. But there is more: even the mere existence of a model with such a code appears denied by the same randomness. In fact it seems impossible to define, by means of a finite number of instructions, arithmetical operation rules valid for unpredictable symbolic sequences.

\section{References}

[1] Gödel, K. (1934) doc. 1934. In: Feferman, S., et al., Kurt Gödel Collected Works, Vol. I, Oxford University Press, Oxford, 350-355.

[2] Craig, W. (1953) On Axiomatizability within a System. The Journal of Symbolic Logic, 18, 30-32. https://doi.org/10.2307/2266324

[3] Vaananen, J. and T. Wang (2015) Internal Categoricity in Arithmetic and Set Theory. Notre Dame Journal of Formal Logic, 56, 122. https://doi.org/10.1215/00294527-2835038

[4] Hintikka, J. (2000) On Gödel. Wadsworth Philosophers Series, 22 p.

[5] Shapiro, S. (2006) Foundations without Foundationalism: A Case for Second-Order Logic. Oxford University Press, Oxford, 87 p.

[6] Henkin L. (1950) Completeness in the Theory of Types. The Journal of Symbolic Logic, 15, 81. https://doi.org/10.2307/2266967

[7] Raguní, G. (2015) Consequences of a Gödel's Misjudgment. Open Access Library Journal, 2, 6-11.

[8] Raguní, G. (2011) Confines lógicos de la Matemática, revista cultural La Torre del Virrey Nexofía. [Logical Boundaries of Mathematics.] 266-280. on the Web: http://www.latorredelvirrey.es/nexofia2011-confines-logicos-de-la-matematica/

[9] Solomonoff, R.J. (1964) A Formal Theory of Inductive Inference. Part I and Part II, Information and Control, 7, 1-22; 224-254.

[10] Kolmogorov, A.N. (1965) Three Approaches to the Quantitative Denition of Information, Problems Inform. Transmission, 1, 1-7.

[11] Chaitin, G.J. (1966) On the Length of Programs for Computing Nite Binary Sequences. Journal of the ACM, 13, 547-569. https://doi.org/10.1145/321356.321363

\footnotetext{
${ }^{9}$ And, of course, it is incapable of generating contradictions.
} 
[12] Chaitin, G.J. (1974) Information-Theoretic Limitations of Formal Systems. Journal of the ACM, 21, 403-424. https://doi.org/10.1145/321832.321839

[13] Chaitin, G.J. (1992) Randomness in Arithmetic and the Decline and Fall of Reductionism in Pure Mathematics. IBM Thomas J. Watson Research Division, New York, $21 \mathrm{p}$.

[14] Raguní, G. (2012) The Gödel's Legacy: Revisiting the Logic. Intellectual Archive, 1, 149-151.

Submit or recommend next manuscript to OALib Journal and we will provide best service for you:

- Publication frequency: Monthly

- 9 subject areas of science, technology and medicine

- Fair and rigorous peer-review system

- Fast publication process

- Article promotion in various social networking sites (LinkedIn, Facebook, Twitter, etc.)

- Maximum dissemination of your research work

Submit Your Paper Online: Click Here to Submit

Or Contact service@oalib.com 